\title{
A High Dynamic Range ASIC for Time of Flight PET with monolithic crystals
}

\author{
Sergio Gómez*, A. Sanmukh \\ Institut d'Estudis Espacials de Catalunya (IEEC), Institut de Ciències Del Cosmos (ICCUB), \\ Universitat de Barcelona (UB) \\ E-mail: sgomez@fqa.ub.edu \\ D. Gascón, D. Sánchez, J. Mauricio, R. Graciani, R. Manera, L. Garrido, J. M. \\ Fernández-Varea \\ Dept. Física Quàntica i Astrofísica, Institut de Ciències Del Cosmos (ICCUB), Universitat de \\ Barcelona (IEEC-UB), Martí i Franquès 1, Barcelona, 08028, Spain (UB) \\ E-mail: dgascon@fqa.ub.edu
}

J. M. Cela, L. Freixas, J. Marín, J. J. Navarrete, J. C. Oller, J. M. Pérez, P. Rato Mendes, O. Vela

CIEMAT, Technology Department

E-mail: jesus.marin@ciemat.es

\begin{abstract}
The HRFlexToT is a 16-channel ASIC for SiPM anode readout designed for Positron Emission Tomography (PET) applications that features high dynamic range ( $>8$ bits), low input impedance, common cathode connection, high speed and low power $(\approx 3.5 \mathrm{~mW} / \mathrm{ch})$. The ASIC has been manufactured using XFAB $0.18 \mu \mathrm{m}$ CMOS technology. The main characteristics of the HRFlexToT, compared to its predecessor, are a new energy measurement readout providing a linear Time Over Threshold (ToT) with an extended dynamic range, lower power consumption and better timing response. Initial measurements show a linearity error below 3\%. Single Photon Time Resolution (SPTR) measurements performed using a Hamamatsu MPPC S13360-3050CS $\left(3 \times 3 \mathrm{~mm}^{2}\right.$ pixel, $50 \mu \mathrm{m}$ cell) shows $30 \%$ improvement with respect to the previous version of the ASIC, setting this specification in the order of $141 \mathrm{ps}$ FWHM and reducing 3 times power consumption. It is important to highlight that an SPTR of 141 ps FWHM is, according to the best of our knowledge, the best resolution achieved so far for this sensor. Coincidence Time Resolution (CTR) measurements are expected to be performed during 2018.
\end{abstract}

Topical Workshop on Electronics for Particle Physics (TWEPP2018)

17-21 September 2018

Antwerp, Belgium

${ }^{*}$ Speaker. 


\section{Introduction}

Positron Emission Tomography (PET) is a diagnosis technique that employs an small quantity of a radioactive substance in order to obtain molecular images of a living organism. Advances in PET technology result in regular performance improvements and sensitivity increase. PET can be divided into pre-clinical imaging, organ dedicated imaging and whole-body imaging depending on the application. Spatial resolution is a key parameter for these applications in the performance of the detectors. Pre-clinical and dedicated organ PET imaging request volumetric resolution in the order of $1 \mathrm{~mm}^{3}$. In general, whole body PET imaging can relax this figure. Another motivation for even larger clinical sensitivity is to open the way to a drastic reduction of doses injected to the patient [1]. Thus, the technological implementation of PET requires fast and efficient detectors coupled to fast readout electronics.

This work presents HRFlexToT (High Resolution Flexible Time-over-Threshold) which is a new version of the FlexToT ASIC [2] designed with an extended dynamic range suitable for readout of pixelated and monolithic scintillator crystals in Time-of-Flight (TOF) PET applications [3]. It features 16 channels, high dynamic range, high speed, a low input impedance stage based on a MOSFET current mirror with double feedback loop, common cathode connection, directly coupled input with common-mode voltage control and separated timing and energy measurement output.

This paper is organized as follows. A brief explanation of the new architecture of the HRFlexToT ASIC is detailed in section 2. Section 3 provides preliminary experimental results in terms of time and energy resolution of the chip. Lastly, the paper concludes in section 4 providing some future avenues of research.

\section{HRFlexToT architecture}

The main improvements of the HRFlexToT, over its predecessor, are a totally new energy measurement readout providing a linear ToT with an extended dynamic range, lower power consumption and better timing response, i.e., better SPTR which is directly translated into better spatial resolution using TOF image reconstruction. The HRFlexToT block diagram is shown in Figure 1.

The patented current division scheme [3] at the very front-end part of the circuit splits the input current into differently scaled copies which are connected to independent current mirrors to provide the different functionalities of the ASIC. Time response is achieved by using a leading edge current comparator just after the input current conveyor, which provides a non-linear Time over Threshold (ToT) response encoding the collected charge of the photo-electrons following a pulse width modulation. Note that the main limitation of these kind of ToT comparators is that the relation between the input charge to be measured, i.e., the number of photo-electrons detected, and the width of the encoded pulse is non-linear. A fast OR between all timing channels can be read out using a differential LVDS output and individual time readout is provided in single-ended output.

The energy measurement block (Figure 2) is the main difference between the HRFlexToT and its predecessor. The previous version was composed by an RC integrator and a constant DC current source. The linear response was achieved by initially charging a capacitor with the incoming signal, then discharging it using a constant DC current and thus providing a linear discharge and lastly feeding this signal into a voltage discriminator. The problem of this method is that for low currents, 


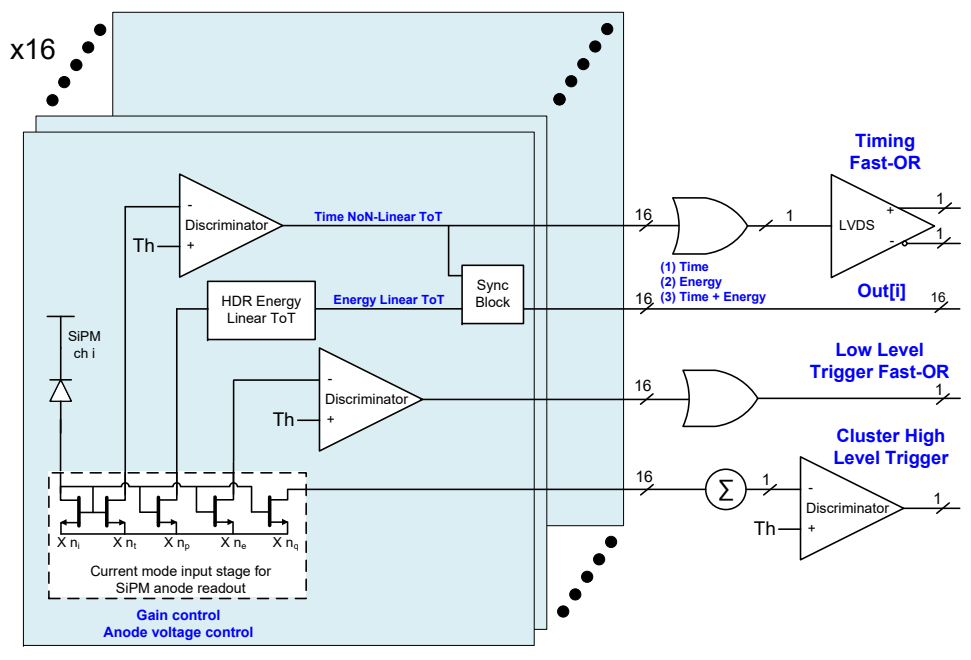

Figure 1: Functional block diagram of the HRFlexToT ASIC.

the discharge of the capacitor stops behaving linearly since part of the DC current starts flowing through the feedback network of the RC integrator instead of discharging the capacitor. This effect for low currents is translated into a reduction in the linearity and thus reducing the dynamic range (input peak current range is from around $2.5 \mathrm{~mA}$ to at least $18 \mathrm{~mA}$ ).

The new redesigned energy path, which is conceptually similar to a Wilkinson ADC, does not suffer the problem of low currents. It provides a linear ToT output encoding the collected charge in a binary pulse width with an extended dynamic range (input peak current range is from 150-200 $\mu \mathrm{A}$ to at least $35 \mathrm{~mA}$ ) and allowing a resolution higher than 8 bits. The main increase in terms of dynamic range is achieved by increasing the minimum current that provides a linear energy response. Hence, this new version can distinguish pulses close to the level of the noise, which is around $0.5 \mathrm{~ns}$, up to pulses with an amplitude of $500 \mathrm{~ns}$. The increase of the sensitivity at low currents is important with monolithic crystals where signal is spread among all SiPMs in arrays.

The energy measurement is obtained in four stages. The first stage is a trans-impedance amplifier and a semi-Gaussian shaper, which can be configured to cope with different scintillators and sensors providing around 50-80 ns peaking time. The shaper block is implemented using a CR-RC pulse shaping network which contains a passive pole zero cancellation (CR block) and an active integrator (RC block) and provides an output pulse with a nearly Gaussian shape. The purpose of this block is to "shape" the signal by shortening the decay time of the pulse to minimize the effect of after-pulsing, this effect is achieved by the CR filter. Moreover, the RC filter makes the rising edge of the pulse stretched and thus slew rate requirements in subsequent processing stages are less demanding.

Secondly, a ramp generator starts a linear ramp after detecting the incoming signal with a configurable delay larger than the shaper peaking time. Thirdly, the peak is sampled by a peak detector that can be also configured as a track and hold (PDH), which is based on [4]. Lastly, a rail-to-rail comparator generates a linear ToT energy pulse using the peak detected and the ramp signal as a threshold. Hence, this mechanism provides a linear ToT response since the ramp used as threshold is linear and the amplitude of the incoming signal (encoding the collected charge) follows 
a linear relation with respect to the number of photo-electrons detected. Individual control of the threshold at the comparator is available.

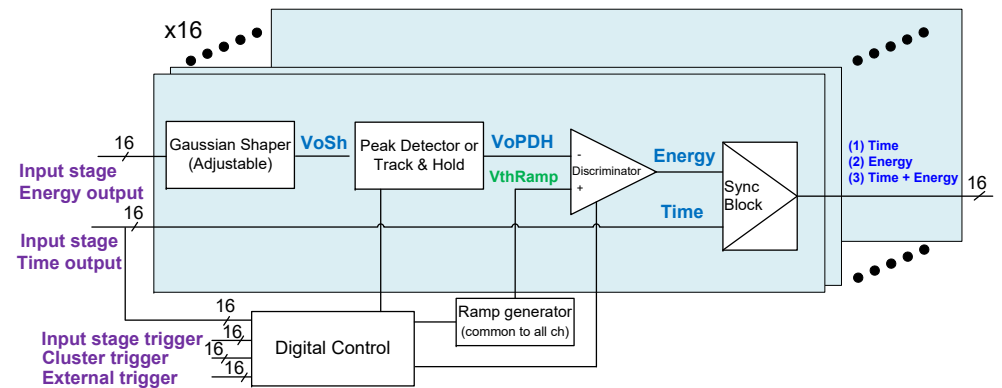

Figure 2: Functional block diagram of the new energy readout scheme.

Different triggers can be used for the energy measurement: a low-level trigger for signals above 10 photo-electrons that allows to filter Dark Count events; the timing signal of the input stage for trigger levels close to the first photo-electron; a cluster high-level or summation trigger (input signal of all channels is added into a single output before the discriminator, as shown in Figure 1) in case signal is spread among several channels; an external trigger for pedestal calibration. The control of the energy acquisition is performed by an asynchronous Finite State Machine (FSM), which drastically reduces cross talk interference of digital signals on the analog circuitry.

\section{Experimental Results}

A considerable reduction in power consumption, from $10 \mathrm{~mW} / \mathrm{ch}$ to $3.5 \mathrm{~mW} / \mathrm{ch}$, is partly achieved by using XFAB $0.18 \mu \mathrm{m}$ CMOS technology instead of AMS $0.35 \mu \mathrm{m}$ HBT BiCMOS technology as was utilized in the FlexToT ASIC. Initial measurements with a Hamamatsu MPPC S13360-3050CS ( $3 \times 3 \mathrm{~mm}^{2}$ pixel, $50 \mu \mathrm{m}$ cell) show a SPTR of 60 ps sigma, i.e., 141 ps FWHM, which is, according to the best of our knowledge, the best resolution achieved so far for this sensor (Figure 3). Note that the SPTR measurements has been performed with a pulsed laser with $635 \mathrm{~nm}$ wavelength and $20 \mathrm{ps}$ jitter.

SPTR measurements performed using a Hamamatsu S13360-3050CS MPPC ( $3 \times 3 \mathrm{~mm}^{2}$ pixel, $50 \mu \mathrm{m}$ cell) shows $30 \%$ improvement with respect to the previous version of the ASIC, setting this specification in the order of 60 ps sigma (141 ps FWHM), as shown in Figure 3 and 4a. Conversion of electrical injected signal (emulating the response of an SiPM) shows a linearity error of the energy measurement around 3\%. Initial energy measurements performed with a $\mathrm{Na}-22$ radiation source shows an energy resolution at $511 \mathrm{keV}$ lower than 10\% (FWHM) (Fig. 4b), although better results are expected in future measurements.

\section{Conclusions and outlook}

Preliminary experimental results of the new generation ASIC of the FlexToT family for PET medical imaging shows a promising performance. The HRFlexToT provides a linear ToT with an extended dynamic range, lower power consumption and better timing response. Future work will include more measurements of SPTR and Coincidence Time Resolution (CTR) measurements. 

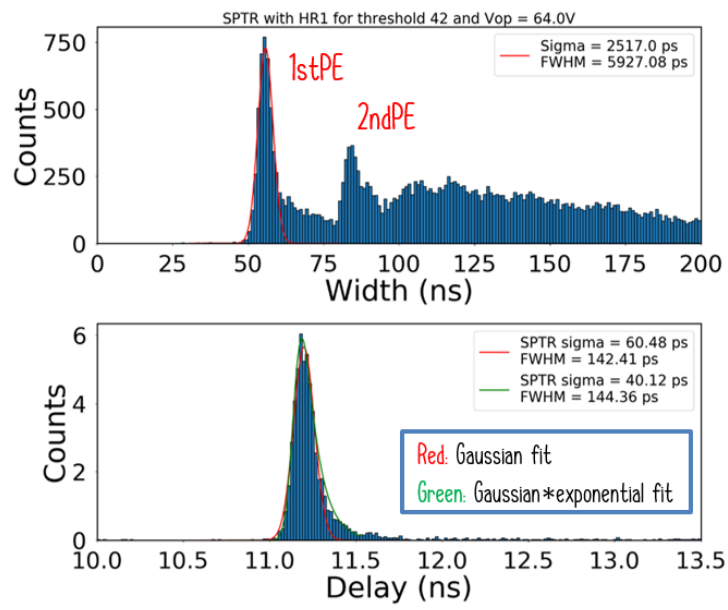

Figure 3: (Above) non-linear ToT width distribution and (bottom) delay of arrival time of the first photon for Time output using Hamamatsu MPPC S13360-3050CS ( $3 \times 3 \mathrm{~mm}^{2}$ pixel, $50 \mu \mathrm{m}$ cell).

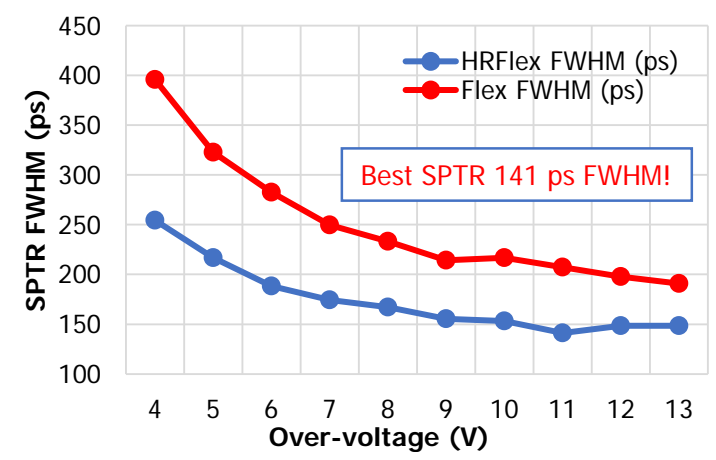

(a) Time resolution (SPTR in terms of FWHM) comparing the FlexToT and HRFlexToT ASICs for different over-voltages.

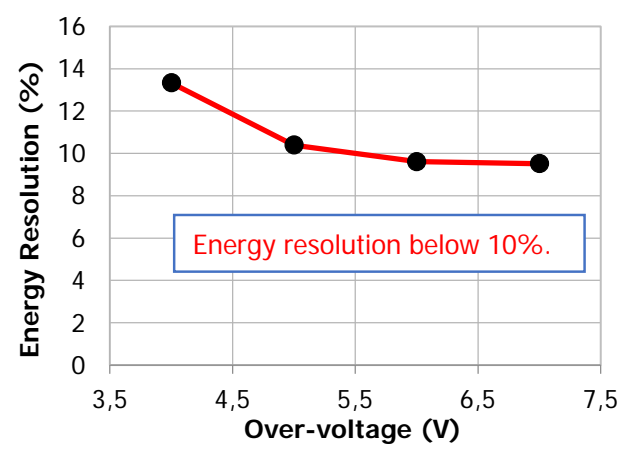

(b) Energy resolution (using FWHM) at 511 $\mathrm{keV}$ using an Na22 source and a LFS scintillator crystal $3 \times 3 \times 20 \mathrm{~mm}^{3}$.

Figure 4: Time and energy resolution using Hamamatsu MPPC S13360-3050CS (3x3 mm² pixel, $50 \mu \mathrm{m}$ cell).

\section{References}

[1] P. Lecoq, Pushing the Limits in Time-of-Flight PET Imaging, IEEE Transactions on Radiation and Plasma Medical Sciences 1 (nov, 2017) 473-485.

[2] J. Trenado, J. M. Cela, A. Comerma, D. Gascon, R. Graciani, L. Freixas et al., Performance of FlexToT time based PET readout ASIC for depth of interaction measurements, in Proceedings of Science (P. (TIPP2014), ed.), 2014.

[3] D. Gascon, A. Comerma and L. Freixas, Readout circuits for multi-channel photomultiplier arrays, European Patent: EP 12382516.8, 2012.

[4] G. De Geronimo, P. O'Connor and A. Kandasamy, Analog CMOS peak detect and hold circuits. Part 2. The two-phase offset-free and derandomizing configuration, Nuclear Instruments and Methods in Physics Research, Section A: Accelerators, Spectrometers, Detectors and Associated Equipment $\mathbf{4 8 4}$ (may, 2002) 544-556. 